\title{
A Revolutionary Approach for Molecular Imaging with TOF-SIMS Parallel Imaging MS/MS
}

John S. Hammond ${ }^{1}$, Gregory L. Fisher ${ }^{1}$, Paul E. Larson ${ }^{1}$ and Scott R. Bryan ${ }^{1}$

1. Physical Electronics USA, Chanhassen, Minnesota, USA

At the time of introduction in the 1980's, TOF-SIMS was a surface analysis research instrument providing elemental ion and limited molecular fragment ion spectra and images of the outer layer of solid materials. Since the initial successes, TOF-SIMS has evolved into a more powerful technique based on several important instrumentation advances. These advances, listed in chronological order, are listed below:

- Improved mass resolution up to $16,000 \mathrm{~m} / \Delta \mathrm{m}$

- Improved depth of field up to $200 \mu \mathrm{m}$ for analysis of many real world samples

- Enhanced higher mass fragment ion sensitivity using cluster ion probe beams, i.e. $\mathrm{Bi}_{\mathrm{n}}{ }^{\mathrm{q}+}$

- Organic depth profiling, with minimal chemical damage, using cluster ion sputter sources $\mathrm{OSF}_{6}$

$\mathrm{OGlycerol}^{+}$

$\circ \mathrm{C}_{60}{ }^{+}$

$\circ$ Coronene $^{+}$

$\circ \mathrm{Ar}_{\mathrm{n}}{ }^{+}$Gas Cluster Ion Beam (GCIB)

- 3D Imaging, FIB-TOF and 3D Tomography

All of these advances have expanded the breadth of applications, most notably the use of TOF-SIMS for analyzing organic contamination, tissue cross sections and polymer surfaces with sub-micron spatial resolution. The limitation of organic molecular fragment compositional peak assignments, based on the mass resolution up to $16,000 \mathrm{~m} / \Delta \mathrm{m}$ and the mass accuracy of $\sim 10$ to $20 \mathrm{ppm}$ for current state-of-the-art TOF analyzers, has obviated some of the benefits of the listed advancements. Routine analysis can produce ion spectra and ion images over $2,000 \mathrm{~m} / \mathrm{z}$. For many molecular ion fragments, unambiguous peak assignments cannot be achieved over a mass of $\sim 200 \mathrm{~m} / \mathrm{z}$ (for either positive or negative polarity ions). Clearly a paradigm shift is needed in the data acquisition of higher mass molecular ion fragments for TOF-SIMS to reach its potential for sub-micron spatial resolution analyses. This is particularly important for cellular and sub-cellular biological tissue analysis, as well as micro-features on polymers, biomaterials, and other organic materials.

To provide this needed paradigm shift, a new TOF-SIMS Parallel Imaging MS/MS instrument has been designed and a review of results from this instrument will be presented. The newly developed TOFTOF tandem imaging mass spectrometer allows conventional TOF-SIMS $\left(\mathrm{MS}^{1}\right)$ analysis and product ion $\left(\mathrm{MS}^{2}\right)$ analysis to be acquired simultaneously and in parallel. Secondary ions for MS ${ }^{1}$ and $\mathrm{MS}^{2}$ analysis are produced from the same area of the surface by a pulsed and digitally raster-scanned primary ion nanoprobe. Activation of the precursor ions, defined by a $1 \mathrm{Da}$ precursor selection window, is accomplished by $1.5 \mathrm{keV}$ CID using Ar gas. Lateral resolutions produced in both $\mathrm{MS}^{1}$ and $\mathrm{MS}^{2}$ images are demonstrated in the present research to be $<200 \mathrm{~nm}$. The $\mathrm{MS}^{2}$ fragment spectra from pure Crystal Violet demonstrates that the 1 dalton wide precursor selection window can eliminate or select ${ }^{13} \mathrm{C}$ containing precursor peaks, providing enhanced MS/MS data from samples with complex mixtures of additives on the surface. This data also bodes well for imaging of lipids and metabolites from tissue samples. The $\mathrm{MS}^{2}$ fragment spectra also demonstrate better than $10 \mathrm{ppm}$ mass accuracy for the identified peaks. The conversion efficiency of precursor ion peaks to the spectrum of fragment ion 
peaks is, on average, $\sim 10 \%$.

The PHI nanoTOF II imaging MS/MS schematic is shown in Figure 1. The system design allows simultaneous MS and MS/MS imaging up to $8.3 \mathrm{kHZ}$. Figure 2 shows the MS/MS spectrum of the Erucamide polymer additive negative polarity precursor molecular ion. The spectrum allows the structural identification of the double bond location. Figure 3 shows the MS images for a heat treated polyethylene terephthalate film and the MS/MS images from the positive polarity $577 \mathrm{~m} / \mathrm{z}$ precursor ions. The simultaneous $\mathrm{MS}^{1}$ and $\mathrm{MS}^{2}$ imaging of the heat treated PET sample confirmed that the crystal structure on the surface of the PET is a cyclic trimer structure. The MS ${ }^{1}$ images and $\mathrm{MS}^{2}$ images derived from the CID activation of the positive polarity $577 \mathrm{~m} / \mathrm{z}$ precursor show that the crystal images are in exact registry with one another. Line scans ( $80 \%$ to $20 \%$ intensity) show <200 nm spatial resolution for both modes of imaging.
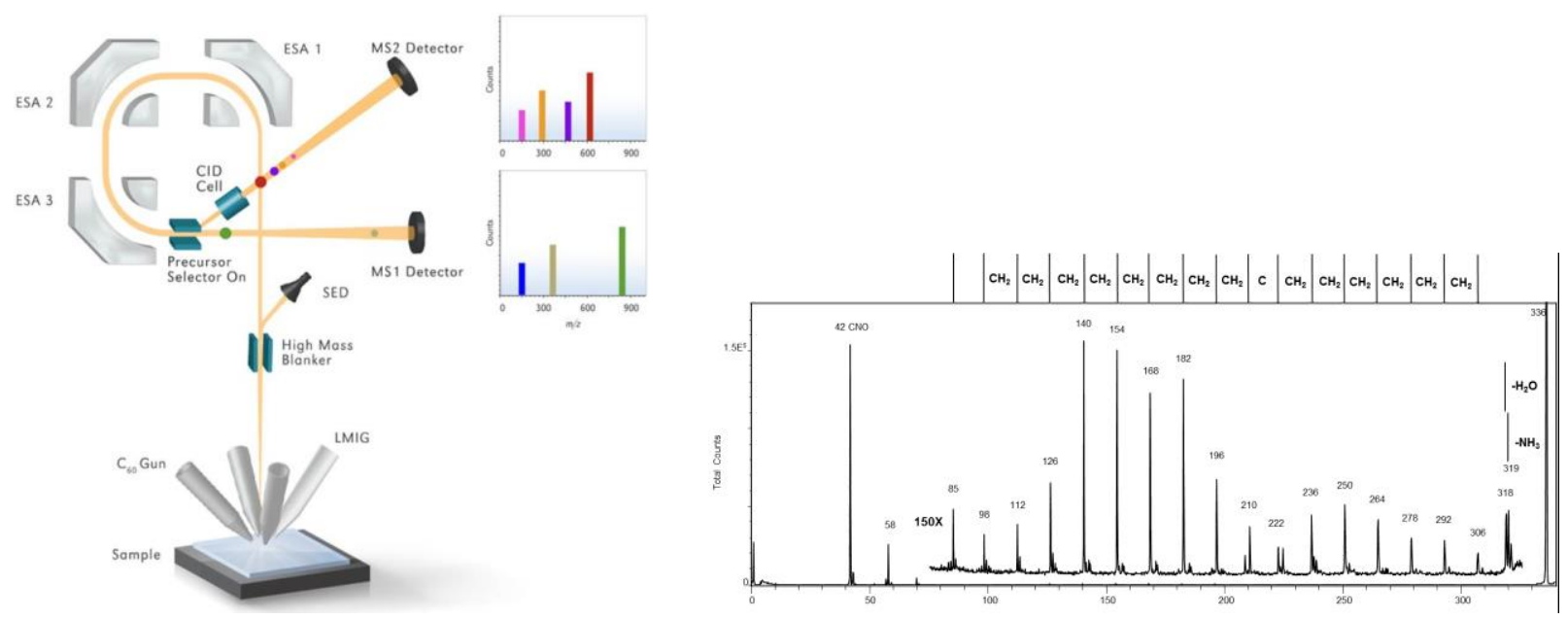

Figure 1. TOF-SIMS Imaging MS/MS ion
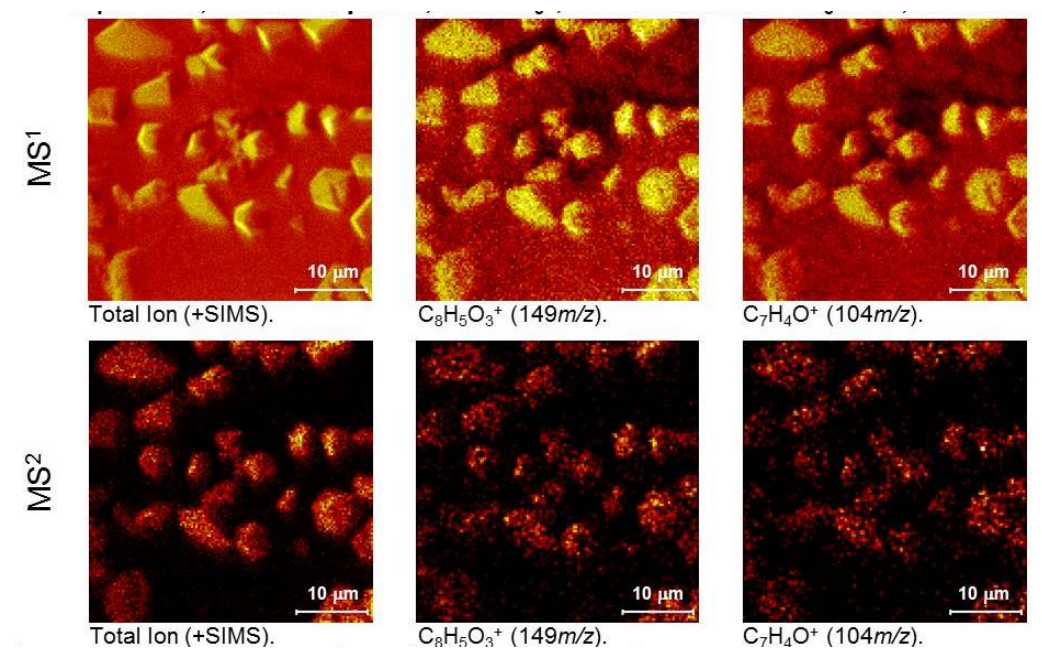

Figure 3. $\mathrm{MS}^{1}$ images, and MS/MS images from positive polarity $577 \mathrm{~m} / \mathrm{z}$ precursor ions, from heat treated PET 